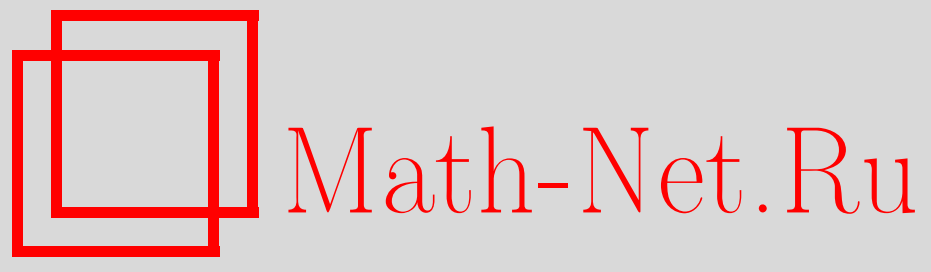

С. Е. Степанов, В. Н. Шелепова, Заметка о солитонах Риччи, Матем. заметки, 2009, том 86, выпуск 3, 474477

DOI: https://doi.org/10.4213/mzm8508

Использование Общероссийского математического портала Math-Net.Ru подразумевает, что вы прочитали и согласны с пользовательским соглашением http://www . mathnet.ru/rus/agreement

Параметры загрузки:

IP : 52.90 .164 .192

26 апреля 2023 г., $17: 47: 40$

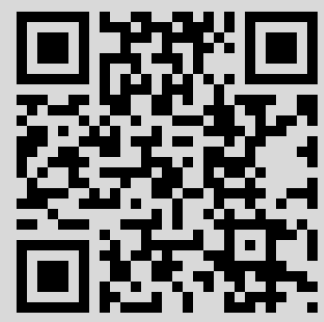




\section{Заметка о солитонах Риччи}

\section{С. Е. Степанов, В. Н. Шелепова}

1. Введение. Определение солитона Риччи принадлежит Гамильтону. Солитон Риччи задается системой уравнений эллиптического типа для солитона и некоторого ассоциированного с ним векторного поля. За исключением случая градиентности, других, сколько-нибудь геометрических свойств этого векторного поля, при изучении солитонов Риччи в литературе не рассматривалось, в противоположность, например, понятию солитона Ямабе в теории потоков Ямабе, определение которого, как и солитона Риччи, связано с векторным, но уже конформно киллинговым полем. В настоящей статье мы сделаем акцент на описании солитонов Риччи за счет геометризации понятия участвующего в его определении векторного поля.

2. Солитоны Риччи. Семейство римановых метрик $g_{t}=g(t)$ на $n$-мерном $(n \geqslant 3)$ гладком многообразии $M$, определенных на включающем нуль временном интервале $J \subset \mathbb{R}$, называется потоком Риччи, если выполняются уравнения Гамильтона потока Риччи (см. [1; с. 21], [2; с. 98])

$$
\frac{\partial g}{\partial t}=-2 \text { Ric }_{0}
$$

для $g_{0}=g(0)$ и тензора Риччи Rico метрики $g_{0}$. C самоподобным решением уравнений (2.1) связано понятие солитона Риччи (см. [1; с. 22-23]), как метрики $g_{0}$, удовлетворяющей уравнениям

$$
-2 \mathrm{Ric}_{0}=L_{X_{0}} g_{0}+2 \lambda g_{0}
$$

для некоторого векторного поля $X_{0}$ на $M$, производной Ли $L_{X_{0}}$ по отношению к $X_{0}$ и постоянной $\lambda$. Для $\lambda=0$ солитон Риччи называется устойчивым, для $\lambda<0$ - стягивающимся и, наконец, для $\lambda>0$ - растягивающимся. Метрика $g_{0}$ называется градиентным солитоном Риччи (см. [1; с. 22], [2; с. 154]), если $X_{0}$ является градиентом некоторой функции $f$.

3. Основные результаты. Векторное поле $X$ на римановом многообразии $\left(M, g_{0}\right)$ называется инфинитезималъным гармоническим преобразованием, если индуцированная им 1-параметрическая группа локальных преобразований многообразия $\left(M, g_{0}\right)$ состоит из локальных гармонических диффеоморфизмов (см. [3], [4]). Справедлива

Теорема 1. Векторное поле $X_{0}$ на многообразии $M$, ассоциированное с солитоном Риччи $g_{0}$, является инфинитезимальным гармоническим преобразованием на римановом многообразии $\left(M, g_{0}\right)$.

Как это показано в [3], инфинитезимальные гармонические преобразования на римановом многообразии $\left(M, g_{0}\right)$ составляют ядро лапласиана Яно (см. [5; с. 40]). На основании этого факта доказывается

СлЕДСтвиЕ 1. На компактном многообразии $M$ не существует солитона Риччи $g_{0}$ и ассочиированного с ним векторного поля $X_{0}$ таких, что $\operatorname{Ric}_{0}\left(X_{0}, X_{0}\right)<0$.

Заметим, что в следствии 1 условие $\operatorname{Ric}_{0}\left(X_{0}, X_{0}\right)<0$ можно заменить более жестким требованием $\operatorname{Ric}_{0}<0$, в результате чего $X_{0} \equiv 0$ и растягивающийся солитон становится тривиальным, т.е. $\mathrm{Ric}_{0}=-\lambda g_{0}$. Из теоремы 1 также выводится

СледствиЕ 2. На компактном многообразии $M$ не существует нетривиальных растягивающихся солитонов Риччи.

(C) С. Е. СтепАнов, В.Н. ШЕлЕпова, 2009 
Напомним, что конформно киллинговое векторное поле $X$ на римановом многообразии $\left(M, g_{0}\right)$ индуцирует 1-параметрическую группу локальных конформных диффеоморфизмов $\left(M, g_{0}\right)$ и задается уравнением

$$
L_{X} g_{0}=2 n^{-1}(\operatorname{div} X) g_{0}
$$

(см., например, [2; с. 516], [6; глава II, §6]). Если подобное поле $X$ является инфинитезимальным гармоническим преобразованием, то $\operatorname{div} X=$ const при $n>2$ (см. [4]). Справедлива также

Лемма 1. Векторное поле $X_{0}$, ассоциированное с солитоном Риччи $g_{0}$, будет конформно киллинговым тогда и толъко тогда, когда gо будет метрикой Эйнштейна.

Для получения характеристики стягивающихся солитонов Риччи докажем сначала, что справедлива

Теорема 2. Если на компактном многообразии $M$ скалярная кривизна $s_{0}$ солитона Риччи $g_{0}$ удовлетворяет условию $d s_{0}\left(X_{0}\right) \leqslant 0$ для ассочиированного с $g_{0}$ поля $X_{0}$, то $g_{0}$ будет метрикой Эйнштейна.

На основании леммы из теоремы 2 выводится

СлЕДСТвИЕ 3. Если на 3-мерном односвязном компактном многообразии $M$ скалярная кривизна $s_{0}$ стягивающегося солитона Риччи $g_{0}$ удовлетворяет условию $d s_{0}\left(X_{0}\right) \leqslant 0$ для ассоциированного с $g_{0}$ векторного поля $X_{0}$, то риманово многообразие $\left(M, g_{0}\right)$ будет изометричным сфере $\mathbb{S}^{3}$.

Заметим, что и в текстах теоремы 2 и следствия 3 условие $d s_{0}\left(X_{0}\right) \leqslant 0$ можно заменить более жестким требованием $s_{0}=$ const.

4. Доказательство утверждений. В локальных координатах $x^{1}, \ldots, x^{n}$ многообразия $M$ уравнения солитона Риччи примут вид

$$
-2 R_{i j}=\nabla_{i} X_{j}+\nabla_{j} X_{i}+2 \lambda g_{i j}
$$

Здесь $X_{i}=g_{i j} X^{j}, R_{i j}$ и $g_{i j}$ - компоненты векторного поля $X_{0}$, тензоров Риччи Ric 0 и $g_{0}$, a $\nabla_{k}$ - символ ковариантного дифференцирования в направлении $\partial_{k}=\partial / \partial x^{k}$, в записи которых символ “0” мы для упрощения обозначений опускаем.

Сверткой с $g^{i j}$ из (4.1) получаем для $X_{0}$ выражение его дивергенции

$$
\operatorname{div} X_{0}:=\nabla_{i} X^{i}=-\left(s_{0}+n \lambda\right),
$$

где $s_{0}=g^{i j} R_{i j}$ - скалярная кривизна метрики $g_{i j}$. Благодаря (4.2), уравнениям (4.1) можно придать вид

$$
2\left(n^{-1} s_{0} g_{i j}-R_{i j}\right)=\nabla_{i} X_{j}+\nabla_{j} X_{i}-2 n^{-1}(\operatorname{div} X) g_{i j} .
$$

Осталось напомнить, что $g_{0}$ является метрикой Эйнштейна (см. [7; с. 65]), если Ric $0=$ $\mu \cdot g_{0}$, где, как нетрудно установить, $\mu=n^{-1} s_{0}$. Лемма доказана.

Ковариантным образом продифференцируем обе части (4.1) в направлении $\partial_{k}=\partial / \partial x^{k}$, а затем проведя альтернацию, получим

$$
2\left(\nabla_{i} R_{k j}-\nabla_{k} R_{i j}\right)=\nabla_{k} \nabla_{i} X_{j}-\nabla_{i} \nabla_{k} X_{j}+\nabla_{k} \nabla_{j} X_{i}-\nabla_{i} \nabla_{j} X_{k} .
$$

Воспользуемся в (4.3) тождеством Риччи $\nabla_{k} \nabla_{i} X_{j}-\nabla_{i} \nabla_{k} X_{j}=-X_{l} R_{j k i}^{l}$, затем свернем обе части полученного равенства с $g^{i j}$ и, наконец, прибегнем к тождеству $2 \nabla_{l} R_{j}^{l}=\nabla_{j} s_{0}$; в итоге получим

$$
-\nabla_{k} s_{0}=-X_{l} R_{k}^{l}+\nabla_{k} \nabla_{l} X^{l}-g^{i j} \nabla_{i} \nabla_{j} X_{k} .
$$


Из (4.2) последует равенство $-\nabla_{k} s_{0}=\nabla_{k} \nabla_{l} X^{l}$, которое позволяет представить (4.4) в следующем виде:

$$
g^{i j} \nabla_{i} \nabla_{j} X^{k}+R_{l}^{k} X^{l}=0
$$

где

$$
\square X^{k}=-\left(g^{i j} \nabla_{i} \nabla_{j} X^{k}+R_{l}^{k} X^{l}\right)
$$

- оператор Яно (см. [5; с.40]), который, как это доказано в [3], является лапласианом. Теорема 1 доказана.

Всюду далее будем предполагать, что $M$ - компактное многообразие. Вслед за Номидзу (см. [5; с. 63]), рассматривая для $M$ его ориентируемое двулистное накрытие, воспользуемся интегральной формулой

$$
\int_{M}\left(\left(g^{i j} \nabla_{i} \nabla_{j} X^{k}\right) X_{k}+|\nabla X|^{2}\right) d V=0,
$$

справедливой для любого векторного поля $X$ на $M$ (см. также [8; гл. II, $\S 1$, формула (1.2)]). Данной формуле в соответствие с (4.5) придадим вид

$$
\int_{M}\left((g \square X, X)+\operatorname{Ric}(X, X)-|\nabla X|^{2}\right) d V=0 .
$$

Теперь очевидно, что для солитона Риччи $g_{0}$ условие $\operatorname{Ric}_{0}\left(X_{0}, X_{0}\right)<0$ несовместимо с интегральным уравнением (4.6). Следствие 1 доказано.

Приведем теперь два известных факта. С одной стороны (см. [6; § 2, пункт 2.3], [9]), на компактном многообразии $M$ растягивающийся солитон Риччи является градиентным. С другой стороны, доказано (см. [7], [9]), что на компактном многообразии $M$ градиентный растягивающийся солитон Риччи $g_{0}$ является метрикой Эйнштейна. В результате (см. также [9]), на компактном многообразии $M$ растягивающийся солитон Риччи является метрикой Эйнштейна. Но для метрики Эйнштейна $g_{0}$ ее скалярная кривизна $s_{0}=$ const при $n \geqslant 3$ (см. [10; с.66]). В этом случае из (4.2) последует $\nabla_{i} X^{i}=$ $-\left(s_{0}+n \lambda\right)=$ const, что для компактного риманова многообразия $\left(M, g_{0}\right)$ в силу теоремы Грина $\int_{M}\left(\nabla_{i} X^{i}\right) d V=0$ означает $s_{0}=-n \lambda$. Тогда для растягивающего солитона Риччи $g_{0}$ его скалярная кривизна $s_{0}<0$ и, следовательно, $\operatorname{Ric}_{0}\left(X_{0}, X_{0}\right)=n^{-1} s_{0}\left|X_{0}\right|^{2} \leqslant 0$. Отсюда с учетом предыдущего следствия выводится справедливость следствия 2.

Для доказательства теоремы 2 воспользуемся интегральной формулой (см. [8; глава II, $\S 1$, формула (1.14)])

$$
\int_{M}\left(g\left(\square X-n^{-1}(n-2) d(\operatorname{div} X), X\right)-2^{-1}\left|L_{X} g-2 n^{-1}(\operatorname{div} X) g\right|^{2}\right) d V=0 .
$$

Для солитона Риччи $g_{0}$ эта формула принимает вид

$$
\int_{M}\left(n^{-1}(n-2) g_{0}\left(d s_{0}, X_{0}\right)-2^{-1}\left|L_{X_{0}} g-2 n^{-1}\left(\operatorname{div} X_{0}\right) g_{0}\right|^{2}\right) d V=0 .
$$

Если предположить, что $g_{0}\left(d s_{0}, X_{0}\right):=d s_{0}\left(X_{0}\right) \leqslant 0$, или, в частности, что $s_{0}=$ const, то из (4.7) последует, что $L_{X_{0}} g=2 n^{-1}\left(\operatorname{div} X_{0}\right) g_{0}$. С учетом этого на основании леммы заключаем, что солитон Риччи $g_{0}-$ метрика Эйнштейна. Если $n=3$, многообразие $M$ односвязное и $g_{0}$ - стягивающийся солитон Риччи, то $\left(M, g_{0}\right)$ будет римановым многообразием постоянной кривизны $K=[n(n-1)]^{-1} s_{0}>0$ (см. [10; с. 66$\left.]\right)$, изометричным cфepe $\mathbb{S}^{3}$. 


\section{СПИСОК ЦИТИРОВАННОЙ ЛИТЕРАТУРЫ}

[1] B. Chow, D. Knopf, The Ricci Flow: an Introduction, Math. Surveys Monogr., 110, Amer. Math. Soc., Providence, RI, 2004. [2] B. Chow, P. Lu, L. Ni, Hamilton's Ricci Flow, Grad. Stud. Math., 77, Amer. Math. Soc., Providence, RI, 2006. [3] S. E. Stepanov, I. G. Shandra, Ann. Global Anal. Geom., 24:3 (2003), 291-299. [4] С. Е. Степанов, И.Г. Шандра, Алгебра и анализ, 16:2 (2004), 154-171. [5] Ш. Кобаяси, Группъ преобразований в дифференциальной геометрии, Наука, M., 1986. [6] G. Perelman, The entropy formula for the Ricci flow and its geometric applications, arXiv: math.DG/0211159v1. [7] R. S. Hamilton, Surveys in Differential Geometry. II (Cambridge, MA, 1993), Cambridge Int. Press, Cambridge, 1995, 7-136. [8] K. Yano, Integral Formulas in Riemannian Geometry, Pure Appl. Math., 1, Marcel Dekker, New York, 1970. [9] H.-D. Cao, Chinese Ann. Math. Ser. B, 27:2 (2006), 121-142. [10] А. Бессе, Многообразия Эйнштейна, Т. 1, Мир, М., 1990.

С. Е. Степанов

Владимирский государственный педагогический университет

E-mail: stepanov@vtsnet.ru

\section{В. Н. Шелепова}

Владимирский государственный педагогический университет
Поступило

23.05.2007 